Symposium zu Crone, Katja: Identität von Personen. Eine Strukturanalyse des biographischen Selbstverständnisses. Berlin: de Gruyter 2016. X, 219 Seiten. [978-3-11-024650-6]

\title{
Identität von Personen - Repliken auf acht Autor*innen
}

Von Katja Crone (Technische Universität Dortmund)

Die folgenden Überlegungen sind Repliken auf Kommentare zu meinem Buch Identität von Personen. Eine Strukturanalyse des biographischen Selbstverständnisses. ${ }^{1}$ Den acht Autor*innen gilt mein herzlicher Dank für ihre herausfordernde und konstruktive Auseinandersetzung mit Teilen meines Buchs. Ich freue mich sehr über die Möglichkeit, meine Thesen weiter zu durchdenken, zu klären und zu begründen. Besonders möchte ich mich bei Gerson Reuter, Eva Backhaus und Jasper Liptow bedanken, die mich im April 2018 zum Buchsymposion Frankfurt-Gießen eingeladen haben, auf dem wir bereits mündlich intensiv über die Thesen und Argumente meines Buchs diskutiert haben.

\section{Tim Henning: Transtemporale Identität und Bewusstsein}

Tim Hennings Kommentar befasst sich mit der von mir vertretenen These, dass Urteile über die transtemporale Identität von Personen semantische Besonderheiten aufweisen. Diese Besonderheiten hängen meinen Ausführungen zufolge mit der Tatsache zusammen, dass Personen über eine erstpersonale Perspektive verfügen. So entspricht es unserem Alltagsverständnis und praktischen Umgang miteinander, dass wir im Falle von Personen, anders als im Falle von Gegenständen wie Stühlen oder Schiffen, eine eindeutige Antwort auf die Frage fordern, ob es sich bei Person A zu t $t_{1}$ und Person B zu t $t_{2} u m$ dieselbe Person handelt oder nicht (siehe dazu z.B. auch Williams 1970, NidaRümelin 2006). Dieses so genannte Eindeutigkeitspostulat stellt Henning in Frage. Es beruhe, so Henning, auf fehlgeleiteten Alltagsüberzeugungen, denn auch im Alltag würden wir gewisse Unschärfen akzeptieren, etwa in Bezug auf den Lebensanfang und das Lebensende von Personen. Henning wählt das Beispiel der Demenz - und setzt damit implizit voraus, dass die zeitliche Existenz von Personen von ihrer psychischen Kontinuität abhängt. Das Beispiel soll unsere Intuition bestätigen, dass Personen ihre Existenz graduell verlassen und wir insofern Grauzonen hinsichtlich der Existenz von Personen zumindest nicht völlig abwegig finden. Diese Beschreibung ist jedoch problematisch. Sie ist empirisch und begrifflich angreifbar, da sie unterbestimmt ist.

\footnotetext{
${ }^{1}$ Das Buch ist im November 2018 als Paperback erschienen.

(C) 2019 Zeitschrift für philosophische Literatur, lizenziert unter CC-BY-ND-3.0-DE
} 
Warum genau sollten wir annehmen, ein Individuum höre graduell auf zu existieren, wenn es krankheitsbedingt einem psychischen Veränderungsprozess unterworfen ist? Unterstellen wir im Falle von psychischen Veränderungen wirklich eine ,variierende Existenz' des betreffenden Individuums? Wem oder welchem Gegenstand schreiben wir psychische Veränderungen zu, wenn es oder er allmählich aufhört zu existieren? Würde Hennings Beschreibung zutreffen, dann würden wir psychische Veränderungen allgemein mit einem graduellen Verlassen oder Betreten der individuellen Existenz verknüpfen, was wir aber gemeinhin nicht tun. Hennings Einwand kann daher nicht überzeugen.

Der zweite Punkt von Hennings Kommentar betrifft meine These, dass der Erlebnisstrom, das Erleben von ineinander übergehenden gehaltvollen mentalen Zuständen, mich darüber informiert, als Erfahrungssubjekt über die Zeit hinweg zu existieren. Die Funktion des Erlebnisstroms hängt meinen Ausführungen zufolge nicht davon ab, dass man ein reflexives Selbstverständnis als Person mit bestimmten Charaktereigenschaften und einer Lebensgeschichte besitzt. Diese Überlegungen stehen im Zusammenhang mit meiner phänomenologisch ausgerichteten Analyse des impliziten Bewusstseins numerischer Identität. Hennings Kritik richtet sich auf die behauptete Unabhängigkeit der einen Form von Subjektivität (zeitlich ausgedehntes Selbsterleben) von der anderen (Selbstverständnis). Dem setzt er eine Kantisch inspirierte Argumentation entgegen, wonach der Bewusstseinsstrom, in dem ich mich als persistierendes Subjekt erlebe, voraussetze, „dass wir uns als äußere Gegenstände begreifen können, die in kausalen Beziehungen stehen“. Um eine Sequenz mentaler Gehalte überhaupt als solche erfassen zu können, müsse ,ich mir meine Erfahrungen als kausal abhängig von der kausalen Folge der wahrgenommenen Ereignisse denken“. Ein Verständnis von uns selbst als in das kausale Weltgeschehen eingebunden liege demnach einem erlebten Bewusstseinsstrom zugrunde. Henning bietet hier eine transzendentale Argumentation an, indem er eine bestimmte Selbstkonzeption für die Möglichkeit eines gegebenen Phänomens, des Bewusstseinsstroms, als notwendig ausweisen will. Meine Argumentation verfährt an dieser Stelle dagegen phänomenologisch, da sie die Struktur eines Bewusstseinsphänomens in den Blick nimmt, worin sich - bedingt durch die subjektive Zugehörigkeit mentaler Zustände eine minimale Subjektivität bemerkbar macht (dass sich hieraus kein Wissen meiner transtemporalen Identität ableiten lässt, soll damit nicht behauptet werden, was ich an verschiedenen Stellen des Buchs deutlich gemacht habe). Mir scheint, dass durch Hennings Einwand verschiedene Argumentations- 
ebenen mit jeweils unterschiedlichen Argumentationszielen vermischt werden. Die von Henning ausgewiesene transzendentale Bedingung kann bereits aus theoretischen Gründen nicht auf der Ebene des gegebenen Phänomens angesiedelt sein. So wird Henning auch nicht behaupten wollen, dass ich - im Zuge meines erlebten Bewusstseinsstroms - eine Selbstkonzeption als in Kausalbeziehungen eingebettetes Wesen gedanklich vollziehen muss; dies wäre auch phänomenologisch inadäquat. Der Blick auf transzendentale Bedingungen ist daher für eine möglichst präzise Phänomenbeschreibung in diesem Fall nicht von Nutzen. Aus diesem Grund kommt eine phänomenologische Analyse des Bewusstseinsstroms, in dem eine Form der minimalen Subjektivität erfahrbar wird, ohne transzendentalphilosophische Begründung aus.

\section{Gerson Reuter: Vorreflexives Selbstbewusstsein: Warum sollte es so et- was geben?}

Gerson Reuters Beitrag befasst sich mit einem der Argumente, die sich in meinem Buch an der Schnittstelle zwischen dem reflexiven und präreflexiven Selbstbewusstsein befinden. Die Argumente dienen dazu, ein explanatorisches Defizit aufzuzeigen. Meine These ist, dass Analysen eines reflexiven Selbstbewusstseins, verstanden als die Fähigkeit zu so genannten Ich-Gedanken, die sich sprachlich mithilfe von so genannten Ich-Äußerungen wieder geben lassen, einer weiteren Begründung bedürfen. Diese These motiviere ich auf dreierlei Weise: durch das Aufzeigen von phänomenologischen Bedingungen, die implizit bereits in Sydney Shoemakers Argument eine Rolle spielen (Shoemaker 1968); im Rückgriff auf das von José Luis Bermúdez aufgeworfene „Paradox des Selbstbewusstseins" (Bermúdez 1998); und anhand eines Zirkeleinwandes, den Fichte gegen das so genannte Reflexionsmodell des Selbstbewusstseins erhebt und der durch Dieter Henrich (1967) berühmt geworden ist. Reuters Kommentar konzentriert sich auf den letztgenannten Punkt. Er geht der Frage nach, was Henrich der Reflexionstheorie des Selbstbewusstseins eigentlich genau vorwirft und ob der Einwand stichhaltig ist. Da der Wortlaut bei Henrich nicht eindeutig und mein Hinweis auf das Argument eher knapp gehalten ist, schlägt Reuter verschiedene Interpretationsmöglichkeiten vor und zeigt die damit verbundenen jeweiligen Probleme auf. Reuters Überlegungen finde ich insgesamt überzeugend. In meiner Replik möchte ich mich mit dem am Ende vorgebrachten Interpretationsvorschlag von Henrichs Argument befassen. Reuter glaubt, Henrich baue in das reflexive Selbstbewusstsein eine Erkenntnisleitung ein, die jedoch sachlich unangemessen sei. Einige Zitate legen es Reuter zufolge nahe, dass Henrich einen Akt der Iden- 
tifizierung von Subjekt und Gegenstand (Subjekt) in Anspruch nehme, aus dem er letztlich seinen Zirkelverdacht ableite. Ich stimme Reuters sachlicher Kritik voll und ganz zu: Es scheint nicht der Fall zu sein, dass man sich im Denken selbstbewusster Gedanken mithilfe eines kognitiven Aktes der Tatsache versichern muss, dass man gerade über sich selbst nachdenkt und einen entsprechenden Akt der Selbstidentifikation vollziehen muss. Die Beschreibung sei sachlich unangemessen, da man im Denken selbstbewusster Gedanken bereits wisse, dass man sich auf sich selbst beziehe. Auch dem kann ich nur zustimmen. Doch genau in dieser Formulierung manifestiert sich meines Erachtens das, was den Zirkelverdacht - so wie ich ihn verstehe - motiviert. Der Zirkeleinwand richtet sich gegen den Versuch, Selbstbewusstsein (um in Reuters Terminologie zu bleiben: das Denken selbstbewusster Gedanken) im Rückgriff auf die Form der Reflexion zu erklären. Reflexion ist - bildlich gesprochen - ein mentales Sich-Zurückwenden auf sich selbst: das Vollziehen eines selbstbezüglichen Gedankens. Einen selbstbezüglichen Gedanken zu vollziehen heißt, sich als Subjekt der eigenen Gedanken, Gefühle und Empfindungen zu denken. Dies setzt aber offenbar ein Bewusstsein davon voraus, dass es dabei um das Subjekt, das man selbst ist, geht - was nichts anderes als ein selbstbezüglicher Gedanke ist. Das Explanandum ist in dem Explanans also bereits enthalten. Diese Rekonstruktion verzichtet auf eine epistemisch starke Form der Selbstidentifikation, wie Reuter sie bei Henrich sieht. Dennoch zeigt sie Grenzen der Erklärung von Selbstbewusstsein im Rückgriff auf die Form der Reflexion auf. Es muss eine nicht-reflexive Form von Subjektivität in den Blick genommen werden, um Selbstbewusstsein als das Denken selbstbezüglicher Gedanken in nicht-zirkulärer Weise zu erklären. Vielleicht meint Reuter etwas Ähnliches, wenn er sagt, dass man im Denken selbstbewusster Gedanken bereits wisse, dass man sich auf sich selbst beziehe. Ich würde hier nicht von einem „Wissen“ sprechen, sondern von einer impliziten Kenntnis, Subjekt meiner mentalen Zustände inklusive reflexiver Gedanken zu sein; dies ist eine Funktion des präreflexiven Selbstbewusstseins. Bleibt die Frage, ob diese Interpretation mit Henrichs Argumentation kompatibel ist. Bislang war ich davon ausgegangen, dass das der Fall ist. Ich nehme Reuters Überlegungen aber zum Anlass, dies noch einmal zu prüfen.

\section{Oliver Schütze: Warum es keine „Ich“-Gedanken gibt}

Der Kommentar von Oliver Schütze nimmt die sprachanalytischen Argumente zur Selbstreferenzialität, die ich im ersten Teil des Buches präsentiere, in den Blick. Sie geben Aufschluss über besondere Merkmale von Selbstrefe- 
renzen. Allerdings, so meine These, bedürfen diese einer Ergänzung durch phänomenologische Beschreibungen, die in der Lage sind, nicht- oder vorsprachliche Formen von Selbstbezüglichkeit ans Licht zu bringen. Schütze ist der Auffassung, dass in meinen sprachanalytischen Erläuterungen zur Semantik des indexikalischen Ausdrucks ,ich“ nicht klar genug zwischen semantischen Eigenschaften linguistischer Entitäten und psychischen Eigenschaften mentaler Zustände unterschieden werde. Anknüpfungspunkt für Schützes Beobachtung ist das Argument von John Perry zur „essenziellen Indexikalität“ (1979), auf das ich mich in meiner Analyse stütze. Vorab möchte ich ein sachliches Missverständnis aus dem Weg räumen: Schütze weist darauf hin, dass die Eigenschaft der Indexikalität nur sprachlichen Ausdrücken wie „hier““, „jetzt“" „heute“, „dort“ und ,ich“" zukommen kann, nicht aber Gedanken (ob nun im Fregeschen Sinn oder als psychische Entitäten verstanden). Dem ist natürlich zuzustimmen. Wenn in meinen Ausführungen von „Ich-Gedanken“ die Rede ist, dann sind damit nicht etwa Gedanken gemeint, denen die Eigenschaft der Indexikalität zukommt, sondern Gedanken, die eine Selbstbezüglichkeit enthalten und die sich sprachlich durch einen Satz wiedergeben lassen, in dem der indexikalische Ausdruck ,ich“ verwendet wird (eine so genannte „Ich-Äußerung“). Dies entspricht der debattentypischen Terminologie. Die damit verbundene methodologische Überlegung ist, dass der Blick auf die Merkmale solcher Äußerungen Hinweise geben auf die Besonderheit von mentaler Selbstbezüglichkeit - im Sinne eines semantic ascents.

Der eigentliche Einwand von Schütze richtet sich gegen die von Perry stammende Auffassung, dass der indexikalische Ausdruck „ich“ in seiner Bedeutung basal ist. Dies besagt, dass die Bedeutung eines Satzes, der den indexikalischen Ausdruck ,ich“ enthält, sich nicht mithilfe eines Satzes analysieren lässt, der anstelle von ,ich“ einen koreferenziellen Term enthält. Dass dies zutrifft, zeigt Perry in Bezug auf sein bekanntes Supermarktbeispiel: Das Verhalten der Person, die endlich feststellt, dass sie selbst die Ursache der Zuckerspur ist, kann nur erklärt werden, wenn man ihr eine Überzeugung zuschreibt, die sich durch einen Satz wiedergeben lässt, der das Indexwort „ich“ enthält. Zu beachten ist zunächst, dass in der Analyse - anders als Schütze anzunehmen scheint - die Ebene des Sprachlichen nicht verlassen wird: Es geht um Überzeugungen, die sprachlich zum Ausdruck gebracht werden, und Verhaltenserklärungen, die ebenfalls sprachlich verfasst sind. Schütze bestreitet nun, dass der indexikalische Ausdruck in seiner Bedeutung basal ist und behauptet, ein Eigenname (als Beispiel für einen koreferenziellen Term) würde in gleicher Weise auf die Sprecherin verweisen und daher in exakt der- 
selben Weise zur Bedeutung eines Satzes beitragen wie der indexikalische Ausdruck ,ich“. Als Begründung führt Schütze an, dass ein Eigenname notwendig (in allen möglichen Welten) auf diejenige Person referiert, die mit diesem Namen getauft wurde. Dieser Hinweis berücksichtigt meines Erachtens nicht die Perspektive von Sprecherinnen und Hörern, auf die es Perry aber gerade ankommt. Die Pointe der Überlegungen ist, dass eine Verhaltenserklärung gelingt, wenn die Bedeutung des Satzes, der eine entsprechende Überzeugung zuschreibt, kognitiv angemessen ist. Dies ist nur dann der Fall, wenn der Satz den Ausdruck ,ich“ enthält - es sei denn, man ergänzt die Äußerung um eine Prämisse wie etwa ,ich bin [Eigenname]“ und versteht die Äußerung als Konklusion eines praktischen Schlusses. Dass dies aber bei der Verwendung von ,ich“ nicht erforderlich ist, macht einen wichtigen Unterschied in der Bedeutung beider Sätze erkennbar, die mit dem basalen Status von „ich“ zusammenhängt.

Aus einem weiteren Grund denke ich, dass Schützes Einwand nicht zielführend ist. Gemeint ist Schützes Verweis auf die Funktion von öffentlichen Ausdrücken allgemein, für den Hörer Bezugsobjekte zu identifizieren, die mit den betreffenden Ausdrücken gemeint seien. Entsprechend bestehe die Funktion des indexikalischen Ausdrucks ,ich“ darin, dass der Hörer die Sprecherin, die den Ausdruck benutzt, identifiziert, nicht aber darin, dass die Sprecherin sich auf eine besondere Art selbst identifiziert. Schütze macht mit diesem Einwand eine problematische Voraussetzung, die sich weniger auf die Frage der essenziellen Indexikalität als vielmehr auf die Art und Weise, wie der Ausdruck „ich“ referiert, bezieht: Behauptet wird, dass der Ausdruck „ich“ - ebenso wie alle anderen sprachlichen Ausdrücke - sein Bezugsobjekt fixiert, indem es dieses identifiziert. Gegen diese Auffassung wenden sich jedoch Argumente (z.B. von Wittgenstein 1984, Shoemaker 1968), die zeigen, dass der korrekte Gebrauch des indexikalischen Ausdrucks ,ich“ gerade keine Identifikation voraussetze, entsprechend auch keine Kriterien erfüllt sein müssen, die das Bestehen der Relation sichern. Und genau deswegen ist, so die genannten Argumente, die Relation von „ich“ und Bezugsobjekt gegen den Irrtum durch Fehlidentifikation immun - eben weil im Falle von „ich“ keine Identifikation vorausgesetzt ist. Schütze müsste zeigen, dass diese Argumente fehlgeleitet sind. Er müsste z.B. zeigen, dass es zwischen dem Subjektgebrauch und dem Objektgebrauch von ,ich“ (Wittgenstein 1984: 106) keinen Unterschied gibt, und dass „ich“ und „du“ auf exakt dieselbe Weise referieren. Schützes Einwand basiert also auf einer Voraussetzung, für die zunächst ein eigenes Argument gegeben werden müsste. 


\section{Martina Büttner: Über phänomenologische Einsichten und ihre In- tegration in naturalistische Erklärungsmodelle}

Gegenstand von Martina Büttners Beitrag ist das methodische Anliegen meines Buchs, Einsichten aus der Phänomenologie in die Analyse des Selbstverständnisses von Personen zu integrieren. Entsprechende Überlegungen entwickle ich u.a. im Zusammenhang des sogenannten präreflexiven Selbstbewusstseins im ersten Teil des Buchs. Hier unterscheide ich verschiedene Komponenten des präreflexiven Selbstbewusstseins, etwa die „Meinigkeit“ und die Perspektivität von mentalen Zuständen und das zeitliche Erleben. Diese Komponenten lassen sich funktional hinsichtlich konkreter inhaltlich gehaltvoller mentaler Zustände sowie eines reflektierten Selbstbewusstseins bestimmen. Die von mir genannten Referenzautoren aus der klassischen Phänomenologie sind u.a. Husserl und Sartre, aus der neueren Diskussion z.B. Zahavi, Gallagher, Bermúdez und Baker. Im Fokus von Büttners Beitrag steht mein Verweis auf die klassische Phänomenologie im Lichte meiner These, dass sich phänomenologische Einsichten in einen naturalistischen Theorierahmen integrieren lassen. Ich vertrete die Auffassung, dass sich phänomenologische Beschreibungen theoretisch nutzen lassen, ohne zusätzliche Annahmen der klassischen Phänomenologie zu übernehmen. Hieran äußert Büttner Zweifel. Diese begründet sie damit, dass sich Husserls und Sartres Auffassungen zum präreflexiven Selbstbewusstsein bereits voneinander unterschieden, und dies sei bedingt durch die jeweiligen zugrunde liegenden Annahmen zum intentionalen Bewusstsein. Hierzu ist zunächst zu bemerken, dass meine Hinweise auf Husserl und Sartre eher lose sind. Sie sollen deutlich machen, dass es für bestimmte Aspekte meiner Analyse nicht nur eine zeitgenössische Diskussion, sondern auch historische Vorläufer gibt. Ich erhebe damit nicht Anspruch einer philosophiehistorisch exakten Untersuchung; meine Überlegungen sind vor allem systematisch motiviert. Dass meine Analyse eher dem Ansatz Husserls als dem von Sartre entspricht, ist sicherlich zutreffend. Diese Beobachtung hat meines Erachtens aber keine Konsequenzen für meine systematische Analyse des präreflexiven Selbstbewusstseins als Bedingung von anspruchsvollen kognitiven Selbstbezugnahmen. Büttner müsste zeigen, dass meine Analyse unter Verzicht auf grundlegende Annahmen z.B. der Husserlschen Phänomenologie inkohärent ist. Das lässt sich aus Büttners Betrachtung aber nicht herauslesen. Daher halte ich es auch für grundsätzlich möglich, phänomenologische Beschreibungen losgelöst von transzendentalphilosophischen Annahmen der klassischen Phänomenologie 
zu betrachten und sie in einen naturalistischen Theorierahmen zu integrieren. Diesen Weg schlage ich in meinem Buch ein.

\section{Christian Kietzmann: Selbstbewusstsein und Selbstbestimmung}

Christian Kietzmann befasst sich in seinem Beitrag mit meinen Überlegungen zum präreflexiven Selbstbewusstsein, das ich als eine Bedingung des biographischen Selbstverständnisses verstehe (Teil I des Buches). Darüber hinaus schlägt er eine Ergänzung zu meiner These über die Selbstzuschreibung von Charaktereigenschaften vor, die im dritten Teil des Buchs thematisch ist. In meiner Replik konzentriere ich mich vor allem auf Kietzmanns Kritik zum ersten Teil. Meine dort vertretene These lautet, dass wir die Struktur von selbstbezüglichen Gedanken, ausgedrückt in Sätzen wie „Ich habe Hunger“ oder „Ich bin mir der Tatsache bewusst, dass p“, besser verstehen können, indem wir uns u.a. mit der Semantik des indexikalischen Ausdrucks ,ich“, den damit verbundenen epistemischen Eigenschaften sowie mit präreflexiven minimalen Formen des Selbstbewusstseins befassen. Dabei gehe ich der Frage nach, ob beispielsweise die von Shoemaker (1968) aufgezeigte Irrtumsimmunität durch Fehlidentifikation bezüglich der ersten Person (IEM) im Rückgriff auf subjektive Erlebniseigenschaften weiter erklärt und erläutert werden kann. Kietzmann hat Zweifel, dass eine Erklärung im Rückgriff auf nicht-sprachliche Phänomene gelingen kann. Dabei deutet sich die Möglichkeit einer solchen Erklärung schon bei Shoemaker selbst an: Dieser befasst sich zwar mit sprachlichen Äußerungen, in denen „ich“ an der Subjektstelle steht. Allerdings handelt es sich bei den für den IEM-Status relevanten Prädikaten, so genannte $\mathrm{p}^{*}$-Prädikaten, um sprachliche Ausdrücke, die auf mentale Zustände mit qualitativen Eigenschaften Bezug nehmen, etwa Schmerzen haben, etwas hören, sehen, fühlen, wahrnehmen. Dies wirft die Frage auf, warum es gerade dieser Typ von mentalen Zuständen ist, deren Instanziierung für den IEMStatus verantwortlich ist. Um diese Frage zu beantworten, bedarf es einer strukturellen Beschreibung der genannten Phänomene, nicht aber einer grammatischen Untersuchung, wie Kietzmann es fordert. Der Grund ist, dass phänomenale Eigenschaften von Erlebniszuständen keine linguistischen Eigenschaften sind. Dieser grundlegende Gedanke findet sich auch in Bermúdez’ „Paradox des Selbstbewusstseins“ (1998): Wenn wir die Struktur von selbstbezüglichen Gedanken zirkelfrei erklären wollen, müssen wir eine nichtsprachliche Form des Selbsterlebens voraussetzen. Dass nun diese nichtsprachliche Form des Selbsterlebens (präreflexives Selbstbewusstsein) nicht eine Spielart des von Kietzmann zitierten „Mythos des Gegebenen“ und mit 
den bekannten Problemen konfrontiert ist, scheint mir auf der Hand zu liegen. Kietzmann verwendet offenbar eine recht grobe Begrifflichkeit, indem er beispielsweise die Ausdrücke „präreflexiv“ und „nicht-begrifflich“ in eins setzt und glaubt, es handle sich dabei um (rein) kausale Eigenschaften. In meiner Argumentation benutze ich die Ausdrücke „präreflexiv“ und „,nichtbegrifflich" jedoch nicht synonym. Das halte ich auch für sachangemessen: Ein Zustand ist „präreflexiv“, wenn er nicht im Fokus der Aufmerksamkeit steht und prinzipiell explizit, d.h. zum Gegenstand eines Gedankens gemacht werden kann. Dagegen ist ein mentaler Zustand „nicht-begrifflich“, wenn dessen Gehalt spezifiziert werden kann, ohne beim Subjekt dieses Zustandes entsprechende Begriffe vorauszusetzen. Ein so verstandener nicht-begrifflicher Zustand kann prinzipiell intentional sein (etwa ein nicht-begrifflich strukturierter Wahrnehmungszustand, z.B. Crane 1988), oder er kann präreflexiv sein, woraus folgt, dass die Eigenschaft der Nicht-Begrifflichkeit in der Eigenschaft der Präreflexivität nicht impliziert ist. Darüber hinaus behaupte ich nicht, dass es sich beim präreflexiven Selbstbewusstsein um einen eigenständigen mentalen Zustand handelt, der losgelöst von z.B. gehaltvollen weltbezogenen Gedanken vorkommen könnte. Vielmehr manifestiert sich das präreflexive Selbstbewusstsein auch in bestimmten Funktionen: in der Zugehörigkeit („Meinigkeit“) von eigenen mentalen Zuständen, in der Perspektivität solcher Zustände etc. Kietzmanns Einwand scheint mir damit entkräftet zu sein.

\section{Andreas Müller: Das Eindeutigkeitspostulat, die Perspektive der ersten Person und transtemporale Identität}

Andreas Müllers Beitrag konzentriert sich auf meine Diskussion zum Problem der transtemporalen Identität von Personen (Teil II des Buchs). Wie Müller gleich zu Beginn richtig feststellt, ist es nicht mein Anliegen, eine bestimmte metaphysische Theorie transtemporaler Identität zu verteidigen. Die Kritik von Müller ist allerdings durchweg so konzipiert, als würde ich dieses Ziel dennoch verfolgen. Zwar trifft es zu, dass ich bestimmte Konsequenzen von metaphysischen Theorien transtemporaler Identität diskutiere und problematisiere. Diese Diskussion bezieht sich aber beispielsweise auf reduktionistische Ansätze allgemein und ist insofern nicht auf Theorien psychischer Kontinuität beschränkt, die Müller in seinem Beitrag aber verteidigen möchte. Entsprechend wird meine Replik keine Erwiderung auf das von Müller vorgebrachte Standardargument für Theorien psychischer Kontinuität sein, vielmehr werde ich die Replik nutzen, um meine im Buch skizzierten Überlegun- 
gen zum Begriff und zum Problem der transtemporalen Identität zu präzisieren. Dabei ist es wichtig, das übergreifende Ziel des Buchs im Blick zu behalten: Darin geht es um eine Strukturanalyse des biographischen Selbstverständnisses von Personen - um das Bewusstsein von Personen, als einzigartige Individuen in der Zeit zu existieren. Entsprechend liegt der Fokus in verschiedenen Teilen des Buches darauf, die Besonderheiten der Perspektive der ersten Person - bezogen auf unterschiedliche relevante Gehalte - aufzuzeigen: in Bezug auf eigene mentale Zustände und dispositionale Eigenschaften, auf autobiographische Episoden und auf die eigene Persistenz oder transtemporale Identität. Die Kapitel zur transtemporalen Identität sollen in erster Linie klären, was uns eine alltagsnahe Sichtweise über die letztgenannte Relation verrät. Es entspricht offenbar unserer alltäglichen Sichtweise und unserem praktischen Umgang miteinander, dass wir mit der Frage, ob eine Person über die Zeit hinweg ein und dieselbe ist, bestimmte Forderungen verknüpfen: Wir fordern, dass es sich bei einer früheren und einer späteren Person - egal wie gravierend die zwischenzeitlichen Veränderungen auch sein mögen - entweder um ein und dieselbe Person handelt oder nicht. Dies hängt meinen Überlegungen zufolge besonders mit der Tatsache zusammen, dass Personen über eine bewusste Innenperspektive verfügen und selbstbewusste Wesen sind. Interessant ist, dass sich diese Beschreibung in existierenden metaphysischen Ansätzen zur transtemporalen Identität von Personen kaum wiederfindet. Dafür gibt es zweifellos bedenkenswerte Gründe. Genau das ist Gegenstand meiner Diskussion: Reduktionistische Ansätze sind, wie Müller richtig feststellt, mit dem Eindeutigkeitspostulat inkompatibel, oder sie führen mit dem Postulat zusammen zu unplausiblen Konsequenzen; nicht-reduktionistische Ansätze, von denen manche zwar die Bedeutung der Innenperspektive von Personen betonen, setzen dagegen unplausible dualistische Annahmen voraus. Hinsichtlich einer tragfähigen Theorie transtemporaler Identität haben meine Überlegungen insofern aporetischen Charakter. Sie sollen aber auch als Aufforderung gelesen werden, die Diskussion zur transtemporalen Identität weiter zu führen, indem stärker berücksichtigt wird, was Personen im Kern ausmacht und sie von anderen Dingen unterscheidet.

Wer versucht, das psychische Kriterium personaler Identität zurückzuweisen, hätte sich zweifellos mit den von Müller skizzierten Einwänden auseinanderzusetzen. Widersprüchlich erscheint allerdings Müllers Behauptung am Ende, wonach das Eindeutigkeitspostulat das psychische Kriterium impliziere; letzteres könne daher nicht sinnvoll zurückgewiesen werden, ohne das erste gleich mit aufzugeben. Zuvor hatte Müller jedoch gezeigt, dass reduk- 
tionistische Ansätze mit dem Eindeutigkeitspostulat in Konflikt stehen. Eine Klärung und Begründung dieser Relation wäre meines Erachtens ein interessanter Anknüpfungspunkt für eine weitergehende Diskussion über die transtemporale Identität von Personen.

\section{Jasper Liptow: Die phänomenale Basis unseres transtemporalen Iden- titätsbewusstseins}

Jasper Liptows Beitrag befasst sich mit meiner in Kapitel 7 des Buchs formulierten These, dass ein explizites Bewusstsein, als numerisch identische Person zu einem früheren Zeitpunkt existiert zu haben, ein phänomenales Erleben der eigenen zeitlichen Existenz voraussetzt. Dabei beziehe ich mich zum einen (1.) auf die Eigenschaft der sog. Meinigkeit oder subjektiven Zugehörigkeit mentaler Zustände, zum anderen (2.) auf qualitative Eigenschaften von episodischen Erinnerungen. Liptow kritisiert, dass die jeweiligen Beschreibungen der Eigenschaften Interpretationsspielräume zulassen. Diese möchte ich im Folgenden beseitigen. Zunächst zu (1.): Die Eigenschaft der „Meinigkeit" wurde in den Debatten zum Selbstbewusstsein bislang ausschließlich in Bezug auf einzelne mentale Zustände analysiert. Demnach ist ein konkreter mentaler Zustand, etwa das Sehen eines gelben Kanarienvogels, durch objektbezogene und subjektbezogene Erlebnisqualitäten gekennzeichnet. Der genannte Wahrnehmungszustand besitzt neben einer spezifischen Empfindungskomponente (z.B. dem Farbeindruck) auch die Eigenschaft der erlebten Zugehörigkeit; ich erlebe ihn als meinen mentalen Zustand. Diese Eigenschaft analysiere ich in Bezug auf den Bewusstseinsstrom, den steten Übergang mentaler Zustände und deren zeitliche Erstreckung. Dabei lässt sich zeigen, dass das Erfahrungssubjekt - aufgrund der subjektiven Komponente - sich als persistierend erlebt. Liptow findet diese Beschreibung plausibel, vorausgesetzt, die subjektive Komponente wird als Teil des Gehalts verstanden und nicht als etwas davon Getrenntes. Dem stimme ich alles in allem zu. Nach Liptow vertritt Dan Zahavi, auf den ich mich streckenweise beziehe, aber die Auffassung, dass die subjektive Eigenschaft der Meinigkeit nicht zum Gehalt mentaler Zustände gehört, sondern als bestimmte Gegebenheitsweise zu verstehen sei. Meint Zahavi tatsächlich, dass sich die subjektive Gegebenheitsweise vom Gehalt trennen lässt? Die von Liptow angeführten Zitate bringen dies meines Erachtens weniger klar zum Ausdruck, als Liptow dies meint. In dem zentralen von Liptow zitierten Passus spricht Zahavi von einer „built-in self-reference“, die z.B. phänomenale Zustände besitzen. Dies deutet meines Erachtens auf ein Enthaltensein des Selbsterlebens im Gehalt des betreffen- 
den mentalen Zustandes hin. In einem Artikel, der 2019 erscheint, wird diese Interpretation nochmals unterstrichen. Hier weist Zahavi darauf hin, dass das in der Meinigkeit sich manifestierende minimale Selbsterleben als eine erlebnishafte Dimension aufzufassen ist, die Teil des phänomenalen Bewusstseins und damit Teil des Gehalts ist.

Allerdings ist dieses Selbsterleben - synchron wie diachron - buchstäblich minimal: In diachroner Hinsicht vermittelt es ein Bewusstsein der persistierenden eigenen Erfahrungsperspektive. Inhaltlich gibt dieses Bewusstsein also nicht viel her, aber mehr brauche ich für mein Argument auch nicht.

Einige letzte Bemerkungen zu (2.) - zur Funktion der phänomenalen Eigenschaft episodischer Erinnerungen. Meine - empirische - These lautet, dass die in Studien zur Gedächtnisforschung nachgewiesene Erlebnisqualität von episodischen Erinnerungen Personen über ihre Persistenz informieren kann. Das kann sie deswegen, weil die Erlebnisqualität einen Selbstbezug enthält (,autonoetisches Bewusstsein“). Liptow meint allerdings, dass diese Interpretation nicht zwingend sei. Denn die Erlebnisqualität könnte auch eine Art kontingenter „Marker“ für diesen Typ mentaler Zustand sein - ohne Selbstbezüglichkeit. Aus empirischen Gründen halte ich dies für unplausibel. Patienten, die unter retrograder Amnesie leiden, können Erinnerungen an Ereignisse ihrer eigenen Vergangenheit unter bestimmten Bedingungen abrufen; diese Erinnerungen werden jedoch als „unpersönlich“ beschrieben, sie fühlen sich an wie Inhalte des semantischen Gedächtnisses, etwa historische Ereignisse, von denen man aus Büchern weiß. Ich glaube nicht, dass sich diese Charakterisierung unter Verzicht auf einen Selbstbezug adäquat beschreiben lässt. Doch einmal angenommen, Liptow habe recht und die Erlebnisqualität episodischer Erinnerungen sei ein kontingenter Marker, durch den wir gelernt haben, episodische Erinnerungen von anderen Typen mentaler Zustände zu unterscheiden. Was genau haben wir dann gelernt? Plausiblerweise haben wir im Laufe der Zeit gelernt, dass das Abrufen von bestimmten Erinnerungen mit einem repräsentationalen Gehalt (z.B. das Brennen der Scheune des Nachbarn vor vielen Jahren) zugleich etwas anzeigt, das man mit sich selbst, dem eigenen zeitlich früheren Erleben des repräsentierten Gehalts, verbindet. Diese Beschreibung, sofern sie mit Liptows Annahme eines kontingenten Markers kompatibel ist, widerspricht aber meiner These nicht. Dass die so strukturierte Erlebnisqualität von Erinnerungen eine Informationsquelle für das Erleben der eigenen Persistenz bildet, halte ich für plausibel. 


\section{Eva Backhaus: Zur Interdependenz von Identität und biographischer Narration}

Die Kritik von Eva Backhaus konzentriert sich auf das Verhältnis des transtemporalen Identitätsbewusstseins zu biographischen Selbstzuschreibungen; dieses Verhältnis diskutiere und analysiere ich im dritten Teil des Buchs. Der Einwand von Backhaus lautet, dass sich eine Interdependenz zwischen den beiden Formen des Selbstbezugs nicht begründen lasse, „wenn man Identitätsbewusstsein als in irgendeiner Weise mit numerischer Identität verbunden sieht". Auf dieses Konditional bezieht sich der erste Teil meiner Replik. „Numerische Identität“ ist offensichtlich eine verkürzte Redeweise, doch lässt sich den anschließenden Erläuterungen entnehmen, dass Backhaus mit dem Ausdruck das Problem der transtemporalen personalen Identität meint: mit der Begründung von notwendigen und hinreichenden Bedingungen, die für die Persistenz einer Person erfüllt sein müssen. Das genannte Konditional ist demnach so zu verstehen: Wenn mit meiner Analyse des Identitätsbewusstseins der Anspruch erhoben würde, notwendige und hinreichende Bedingungen für die Persistenz von Personen bereit zu stellen (oder logisch zu implizieren), könnte eine Interdependenz zwischen Identitätsbewusstsein und biographischen Selbstzuschreibungen nicht begründet werden. So lässt sich beispielsweise nicht bestreiten, dass ich mich in meinem Bewusstsein, als numerisch identische Person über die Zeit hinweg zu existieren, täuschen kann. Backhaus hätte mit ihrem Einwand insofern zweifellos recht. Das Konditional trifft aber, wie Backhaus selbst an einigen Stellen vermutet, auf meine Analyse nicht zu. Ich halte den Versuch, die transtemporale Identität einer Person im Rückgriff auf ihr subjektives Erleben zu begründen, für aussichtslos; dies sollte meine Widerlegung des Arguments von Dainton und Bayne unmissverständlich zum Ausdruck bringen. Wie sind die von Backhaus zitierten Passagen also zu verstehen? In meiner Analyse des Identitätsbewusstseins mache ich Gebrauch von der Bedeutung des Begriffs der numerischen Identität: Personen haben ein implizites Bewusstsein, über die Zeit hinweg als ein und dieselben (numerisch identische) Subjekte zu existieren. Ein solches Identitätsbewusstsein zu haben, heißt aber noch lange nicht - wie Backhaus richtig feststellt -, dass die betreffenden Subjekte auch tatsächlich das sind, was sich ihrem Bewusstsein darstellt. Das behaupte ich aber auch nicht. In den von Backhaus zitierten Passagen benutze ich den Ausdruck „numerische Identität" - ebenfalls in einer verkürzten Redeweise - in dem genannten Sinn, was eigentlich durch den entsprechenden Kontext verdeutlicht werden sollte. Dass sich hier Missverständnisse einschleichen können, kann ich jedoch 
nachvollziehen. In einem 2017 veröffentlichten Aufsatz habe ich einige der zentralen Argumente des Buchs zusammengefasst und mein Verständnis des numerischen Identitätsbewusstseins möglicherweise klarer als im Buch vom metaphysischen Problem der transtemporalen Identität der Person abgegrenzt (Crone 2017).

Eine letzte Bemerkung zum analytischen Gewinn, der sich aus den von mir aufgezeigten Interdependenzen von Identitätsbewusstsein und biographischen Selbstzuschreibungen ergibt. Backhaus meint, dieser sei insgesamt eher überschaubar. Dieses Urteil überzeugt mich nicht. Um den Gewinn besser zu erkennen, muss auch der gegenwärtige Diskussionskontext zum Selbstverständnis von Personen berücksichtigt werden. Narrativistische Ansätze behaupten oftmals, das Selbstverständnis von Personen mittels biographischer Selbstzuschreibungen sei entweder dasselbe wie ein Bewusstsein der eigenen transtemporalen numerischen Identität oder es sei gar für die Persistenz von Personen konstitutiv. Diese falsche Einschätzung führt, wie ich zeige, u.a. auf eine begriffliche Vermengung verschiedener Begriffe von Identität zurück. Ein zentrales Argumentationsziel der Interdependenzthesen besteht nun darin, den semantischen Unterschied zwischen den Identitätsbegriffen und zugleich eine sinnvolle Beziehung zwischen den Begriffen und ihren Anwendungen aufzuzeigen. Auf diese Weise soll also nicht nur die Struktur eines komplexen Bewusstseinsphänomens geklärt, sondern auch eine Verwirrung innerhalb der Diskussion zum Thema behoben werden. Je nach Fokus mag man den Ertrag der Analyse unterschiedlich einschätzen, ich halte ihn jedenfalls aus den genannten Gründen für relevant.

\section{Literatur}

Bermúdez, José L. The Paradox of Self-Consciousness, Cambridge/Mass.: MIT Press, 1998.

Crane, Tim. „The Waterfall Illusion“, Analysis 48 (1988), 142-147.

Crone, Katja. „Strukturen der Identität und des Selbstverständnisses von Personen“, Deutsche Zeitschrift für Philosophie 65.1 (2017), 1-15.

Henrich, Dieter. Fichtes ursprüngliche Einsicht. Frankfurt a.M.: Klostermann, 1967.

Nida-Rümelin, Martine. Der Blick von innen. Zur transtemporalen Identität bewusstseinsfähiger Wesen. Frankfurt a. M.: Suhrkamp, 2006.

Perry, John. „The Problem of the Essential Indexical“, Noûs 13.1 (1979), 321.

Shoemaker, Sydney. „Self-Reference and Self-Awareness“, Journal of Philosophy 65.19 (1968), 555-567. 
Williams, Bernard. „The Self and the Future“, The Philosophical Review 79.2 (1970), 161-180.

Wittgenstein, Ludwig. Das Blaue Buch, Werkausgabe Bd. 5. Frankfurt a. M.: Suhrkamp, 1984.

Zahavi, Dan. „Consciousness and (minimal) selfhood: Getting clearer on formeness and mineness" In The Oxford Handbook of the Philosophy of Consciousness, hg. von Uriah Kriegel, Oxford: Oxford University Press, erscheint 2019. 